Article

\title{
Simultaneous Enhancement of Electrical Conductivity and Seebeck Coefficient of [6,6]-Phenyl-C71 Butyric Acid Methyl Ester $\left(\mathrm{PC}_{70} \mathrm{BM}\right)$ by Adding Co-Solvents
}

\author{
Mina Rastegaralam ${ }^{1}$ (D), Changhee Lee ${ }^{1}$ and Urszula Dettlaff-Weglikowska ${ }^{2, *}$ (D) \\ 1 Department of Electrical and Computer Engineering, Inter-University Semiconductor Research Center, \\ Seoul National University, 1 Gwanak-ro, Gwanak-gu, Seoul 08826, Korea; mn.rstgrlm@gmail.com (M.R.); \\ chlee7@snu.ac.kr (C.L.) \\ 2 Materials Science Consulting and Management, 22113 Oststeinbek, Germany \\ * Correspondence: udettlaff.w@gmail.com
}

Received: 8 April 2018; Accepted: 22 May 2018; Published: 26 May 2018

\begin{abstract}
Chemical modification by co-solvents added to [6,6]-Phenyl-C71 butyric acid methyl ester, commonly known as an n-type semiconducting fullerene derivative $\mathrm{PC}_{70} \mathrm{BM}$, is reported to change the electrical and thermoelectric properties of this system. Power factor of the casted $\mathrm{PC}_{70} \mathrm{BM}$ samples achieves values higher than that determined for a variety of organic compounds, including conducting polymers, such as PEDOT:PSS in the pristine form. After chemical functionalization by different solvents, namely $N, N$-Dimethylformamide (DMF), dimethyl sulfoxide (DMSO), N-Methyl-2-pyrrolidone (NMP), acetonitrile (AC), and 1,2-Dichloroethane (DCE), the four-probe in-plane electrical conductivity and Seebeck coefficient measurements indicate a simultaneous increase of the electrical conductivity and the Seebeck coefficient. The observed effect is more pronounced for solvents with a high boiling point, such as $\mathrm{N}, \mathrm{N}$-Dimethylformamide (DMF), dimethyl sulfoxide (DMSO), and N-Methyl-2-pyrrolidone (NMP), than in acetonitrile (AC) and 1,2-Dichloroethane (DCE). We identified the origin of these changes using Hall mobility measurements, which demonstrate enhancement of the $\mathrm{PC}_{70} \mathrm{BM}$ charge carrier mobility upon addition of the corresponding solvents due to the improved packaging of the fullerene compound and chemical interaction with entrapped solvent molecules within the layers.
\end{abstract}

Keywords: electrical conductivity; Seebeck coefficient; power factor; $\mathrm{PC}_{70} \mathrm{BM}$; figure of merit

\section{Introduction}

Thermoelectric materials are very effective at turning a temperature difference directly into electricity. These materials can contribute to both cooling and thermoelectric power generation $[1,2]$. Traditionally, inorganic materials, such as $\mathrm{Bi}_{2} \mathrm{Sb}_{3}, \mathrm{Bi}_{2} \mathrm{Te}_{3}$, and $\mathrm{PbTe}$, have been used for thermoelectric applications [3]. The problems with using these inorganic compounds are high production costs, toxicity, and scarcity of materials [3,4]. To overcome these problems, organic thermoelectric materials have attracted considerable attention due to their advantages, such as non-toxicity, low cost, mechanical flexibility, abundant raw materials, solution processability, and low thermal conductivity [3-7]. To evaluate the efficiency of a thermoelectric system, a dimensionless quantity called thermoelectric figure of merit ZT is applied. Figure of merit is defined as $Z T=S^{2} \sigma T / k$, where $S$ is the Seebeck coefficient, $\sigma$ is the electrical conductivity, $T$ is the absolute temperature, and $k$ is the thermal conductivity. Thermoelectric materials with high efficiency have a high Seebeck coefficient, high electrical conductivity, low thermal conductivity, and therefore high ZT. To design an effective thermoelectric material, it is necessary to increase electrical conductivity and Seebeck coefficient 
simultaneously, while keeping $\mathrm{k}$ constant. However, this is a challenging task as an increase in the number of carriers from doping will sacrifice Seebeck coefficient. One way towards improving ZT is to make use of chemical functionalization that increases mobility in the material, maintaining a constant number of carriers, which in turn leads to improving both electrical conductivity and Seebeck coefficient, according to the equation $\sigma=\mathrm{en} \mu$, where e is the electron charge, $\mathrm{n}$ is the charge carrier density, and $\mu$ is the carrier mobility [8].

To date, the thermoelectric properties of a variety of organics, such as the following conducting polymers, have been studied: poly [3-hexylthiophene] (P3HT), poly [N-90-heptadecanyl-2,7-carbazolealt-5,5-(40,70-di-2-thienyl-20,10,3-benzothiadizole)] (PCDTBT), polyacetylenes, polyaniline, polypyrrole, poly(paraphenylene), poly(p-phenylenevinylene), poly(carbazolenevinylene) derivative, poly(3,4-ethylenedioxythiophene) polystyrene sulfonate (PEDOT/PSS), FBDPPV, and Poly( $\{4,8$-bis [(2-ethylhexyl)oxy] benzo [1,2-b:4,5- $\left.b^{\prime}\right]$ dithiophene-2,6-diyl\}\{3-fluoro-2-[(2-ethylhexyl) carbonyl] thieno[3,4-b] thiophenediyl\}), known as PTB7 [9-17].

$\mathrm{PC}_{70} \mathrm{BM}$ is a fullerene derivative compound showing electron-transporting properties and a potential for a variety of applications in polymer solar cells and organic electronics [18,19]. However, its low electron mobility and low electrical conductivity are limiting factors for application in practical devices. An effective way to increase the electrical conductivity of fullerenes is chemical doping. Compared with the progress made on $\mathrm{p}$-doping, $\mathrm{n}$-doping is lagging due to the difficulties in finding efficient and stable dopants for n-type organic semiconductors.

Here, we report on thermoelectric properties of pristine $\mathrm{PC}_{70} \mathrm{BM}$ and simultaneous enhancement of its electrical conductivity and Seebeck coefficient, and demonstrate a proof of principle for material modification through addition of co-solvents. The following section contains experimental details related to preparation of the samples, determination of their electrical conductivity, Seebeck coefficients, and charge carrier mobility, followed by a discussion of achieved results.

\section{Experimental}

Figure 1 shows the molecular structure of the applied chemical materials and Table 1 shows the boiling points of the solvents. PCBM was purchased from 1-Material. Chlorobenzene (CB), N,N-Dimethylformamide (DMF), Dimethyl Sulfoxide (DMSO), N-Methyl-2-pyrrolidone (NMP), Acetonitrile (AC), and 1,2-Dichloroethane (DCE) were all purchased from Sigma Aldrich (Yongin, Kyungi, Korea) and used as received. Six solutions were prepared by dissolving $40 \mathrm{mg}$ of $\mathrm{PC}_{70} \mathrm{BM}$ in $1 \mathrm{~mL}$ of chlorobenzene with and without adding $0.1 \mathrm{~mL}$ of DMF, DMSO, NMP, DCE, and AC under an argon atmosphere of a glove box. Samples were fabricated by drop casting of the solutions on glass substrates, which were cleaned by sonication in acetone, isopropyl alcohol, and water, and treated with UV ozone for $15 \mathrm{~min}$ before use. The cast films were dried for $24 \mathrm{~h}$ in a glove box. The thicknesses of the samples were measured by the profilometer and were found to be $3.04 \pm 0.06 \mu \mathrm{m}$, on average. The in-plane electrical conductivity was measured for samples by the standard four-probe Van der Pauw method at room temperature. The contacts were placed at the corners of each sample. For comparison the out of plane conductivity of the pristine sample with $1 \mu \mathrm{m}$ thickness was also measured using an electron-only device with the structure shown in Figure 2 . $\mathrm{LiF}$ and $\mathrm{Al}$ electrodes (deposited through a shadow mask) were thermally evaporated in a $\sim 10^{-6}$ Torr vacuum with a $0.5 \mathrm{~nm}$ and $100 \mathrm{~nm}$ thickness, respectively. The Seebeck coefficient was calculated using $\mathrm{S}=-\Delta \mathrm{V} / \Delta \mathrm{T}$, where $\Delta \mathrm{V}$ was the thermoelectric voltage generated along the sample when it was subjected to the temperature difference, $\Delta \mathrm{T}$, as shown schematically in Figure 3. Two K-type thermocouples were used to measure the temperature on both ends of the samples. 


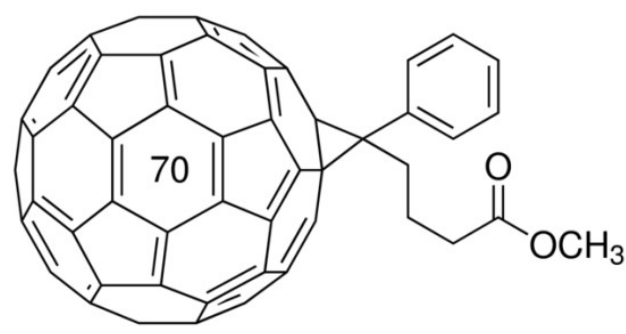

(a)<smiles>CS(C)=O</smiles>

(d)<smiles>Clc1ccccc1</smiles>

(b)<smiles>CN(C)C=O</smiles>

(e)<smiles>CN1CCCC1=O</smiles>

(c)<smiles>CC#N</smiles>

(f)<smiles>ClCCCl</smiles>

(g)

Figure 1. Molecular structure of the applied chemical materials. (a) PCBM; (b) CB; (c) NMP; (d) DMSO; (e) DMF; (f) AC; (g) DCE.

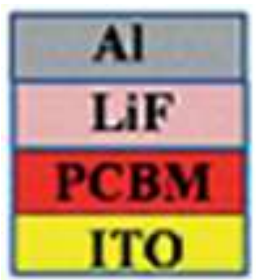

Figure 2. Electron-only device structure.

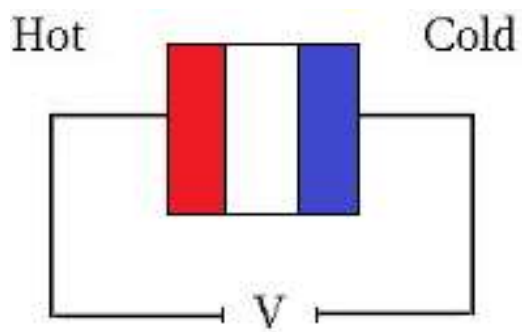

Figure 3. Schematic presentation of the Seebeck coefficient determination.

Table 1. Boiling points of the solvents.

\begin{tabular}{cc}
\hline Material & Boiling Point $\left({ }^{\circ} \mathrm{C}\right)$ \\
\hline Chlorobenzene & 131 \\
1-Methyl-2-pyrrolidone & 202 \\
Dimethylsulfoxide & 189 \\
$N, N-$ Dimethylformamide & 153 \\
1,2-Dichloroethane & 83 \\
Acetonitrile & 82 \\
\hline
\end{tabular}




\section{Results and Discussion}

$\mathrm{PC}_{70} \mathrm{BM}$ can be chemically modified by a variety of chemicals that affect electrical conductivity by doping or de-doping. To find out the effect of co-solvents on the thermoelectric properties of the $\mathrm{PC}_{70} \mathrm{BM}$ samples, a series of solvents with different chemical structures, boiling points, and polarities were selected to be added to the $\mathrm{PC}_{70} \mathrm{BM}$ solution in chlorobenzene for manufacturing samples. Figure 4 reveals the results of in-plane electrical conductivity measurements of the $\mathrm{PC}_{70} \mathrm{BM}$ samples. The diagram shows that the electrical conductivity has increased upon addition of the second solvents. In particular, addition of solvents with higher boiling points leads to larger enhancement of the electrical conductivity by a factor of 3. Considering the boiling point of the solvents, it is clear that the samples in which the solvents with higher boiling points were used need a longer time for drying to complete evaporation. This low evaporation rate allows for better ordering within the molecular packing of the fullerene derivative $\mathrm{PC}_{70} \mathrm{BM}$, and therefore, its ability to transport charge carriers improves, leading to increased electrical conductivity. The best performance was obtained after addition of DMSO. The electrical conductivity values of the samples are $0.108 \mathrm{~S} / \mathrm{m}, 0.135 \mathrm{~S} / \mathrm{m}$, $0.156 \mathrm{~S} / \mathrm{m}, 0.25 \mathrm{~S} / \mathrm{m}, 0.307 \mathrm{~S} / \mathrm{m}$, and $0.323 \mathrm{~S} / \mathrm{m}$ for pristine, CB:DCE, CB:AC, CB:DMF, CB:NMP, and $\mathrm{CB}: \mathrm{DMSO}$ samples, respectively. We anticipated that electrical conductivity of the $\mathrm{PC}_{70} \mathrm{BM}$ will be anisotropic depending on whether the measurement was performed along the layer or perpendicular to it, because the lateral electrical transport depends on the arrangement of fullerene molecules on the plane, while the perpendicular electrical transport is determined by the stacking of individual layers. Indeed, for the $\mathrm{PC}_{70} \mathrm{BM}$ sample, we observed strong anisotropy of conductivity. The out of plane conductivity of the pristine sample was found to be $2.6 \times 10^{-4} \mathrm{~S} / \mathrm{m}$, which is 3 orders of magnitude lower than that of in-plane conductivity. However, for the thermoelectric applications, the in-plane conductivity is essential, as a layered structure is preferential for the multiple p-n junctions connected in series in practical devices.

The effect of chemical modification of $\mathrm{PC}_{70} \mathrm{BM}$ was further investigated by way of measuring the charge carrier mobility in the prepared series of samples. Figure 5 shows that the enhancement of conductivity is accompanied by the increase of the mobility upon adding of the second solvent.

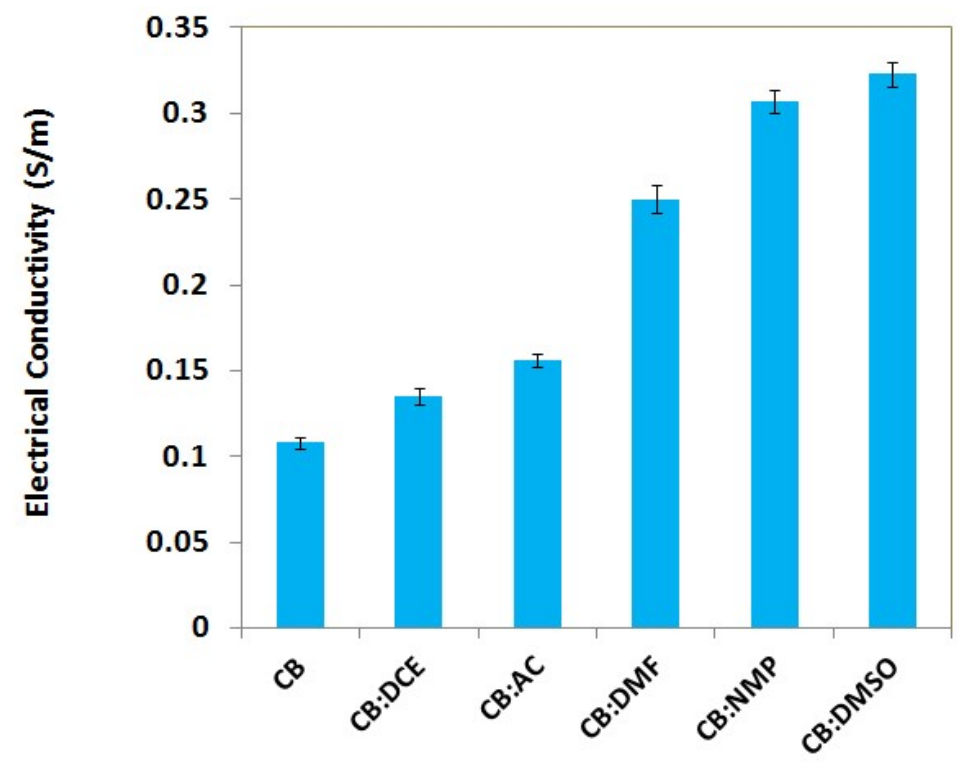

Figure 4. Electrical conductivity of the samples.

The nature of charge carriers in the pristine and treated PCBM samples was determined by the thermoelectric power measurement. The corresponding Seebeck coefficients of the samples are shown in Figure 6. The negative sign of the Seebeck coefficient indicates that the major charge carriers 
are electrons, confirming that all $\mathrm{PC}_{70} \mathrm{BM}$ samples are n-type semiconductor, in pristine form and after treatment by addition of the second solvent. The Seebeck coefficient values of the samples are $-433 \mu \mathrm{V} / \mathrm{K},-440 \mu \mathrm{V} / \mathrm{K},-440 \mu \mathrm{V} / \mathrm{K},-448 \mu \mathrm{V} / \mathrm{K},-461 \mu \mathrm{V} / \mathrm{K}$, and $-464 \mu \mathrm{V} / \mathrm{K}$ for pristine, CB:DCE, $\mathrm{CB}: \mathrm{AC}, \mathrm{CB}: \mathrm{DMF}, \mathrm{CB}: \mathrm{NMP}$, and CB:DMSO samples, respectively.

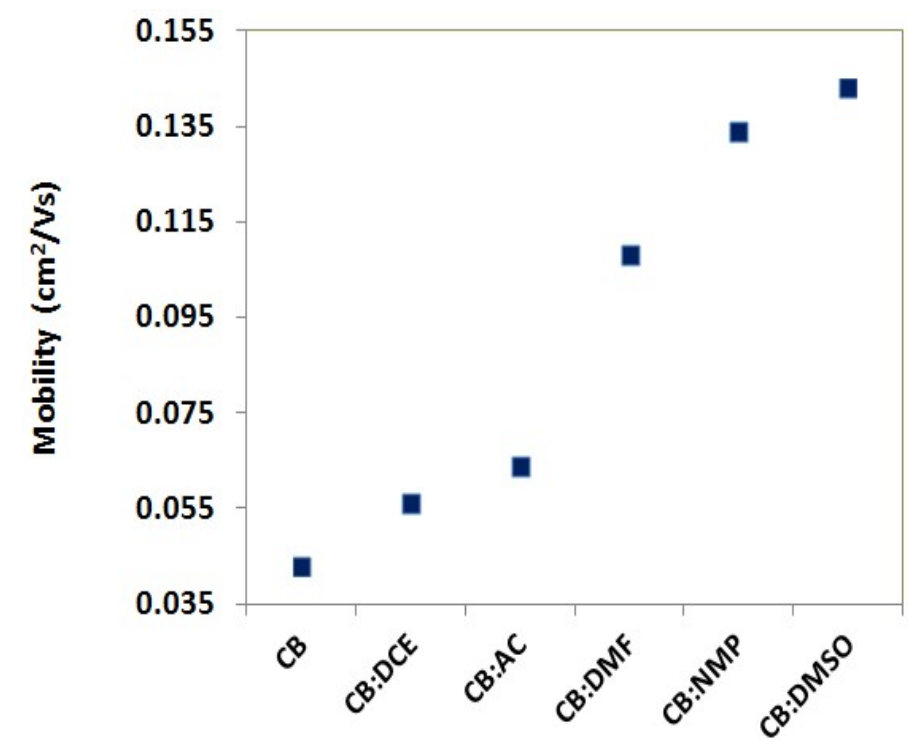

Figure 5. Charge carrier mobility of the samples.

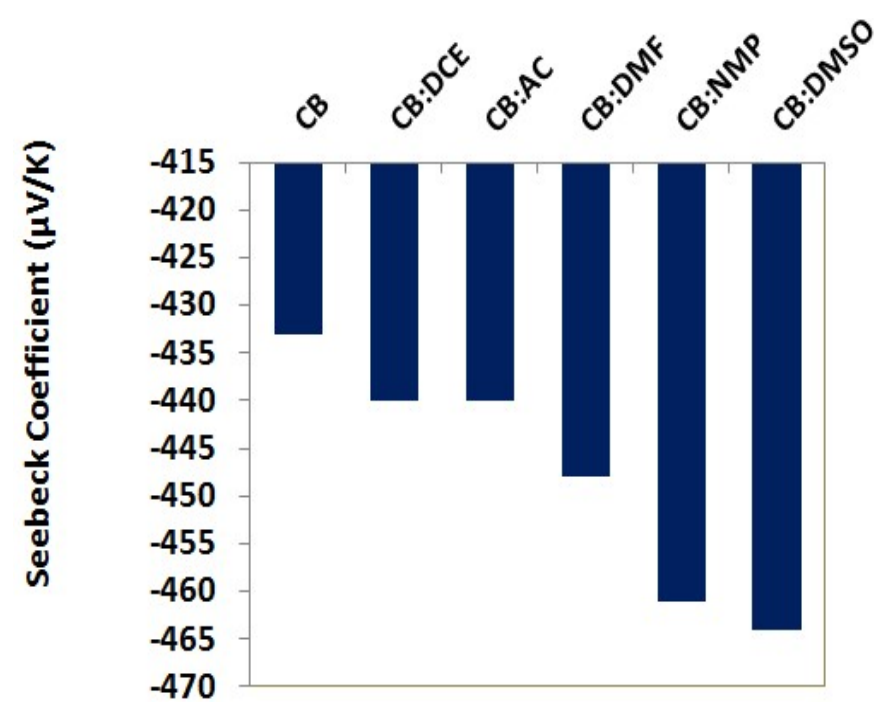

Figure 6. Seebeck coefficient values of the samples.

Previously, it was shown that electrical conductivity increases with increasing boiling point of the used solvent. Similarly, Figure 6 shows that the absolute values of the Seebeck coefficients increase with the increasing boiling point of the solvent as well. The highest Seebeck coefficient was obtained from the CB:DMSO system. Although the applied solvents containing heteroatoms can push or extract electrons from the conducting system, the chemical doping has not been identified within the predefined experimental conditions. Hence, the effect of applying chemical compounds with high boiling points on the thermopower of the $\mathrm{PC}_{70} \mathrm{BM}$ can be explained as follows. While electrical conductivity is associated with the transport of all mobile charges (holes and electrons), the Seebeck coefficient is related to the transport of energetic charges. Transport of charge carriers within the samples under a temperature gradient is described by the heterogeneous model involving highly 
conductive regions separated by barrier regions, such as fullerene inter-junctions and fullerene-solvent interfaces, by a hopping mechanism [13]. Increasing the number of nano-scale barriers in the form of interfaces in heterogeneous materials, such as the investigated arrangement of fullerene derivative network, interacting with the trapped chemical molecules of intentionally added solvents within the film is expected to enhance the thermopower. Therefore, we observed an enhancement of Seebeck coefficient for samples including DCE, AC, DMF, NMP, and DMSO.

The increase of Seebeck coefficient and conductivity in parallel in a hole-conducting system has been demonstrated previously for some doping levels of intentionally doped poly(alkylthiophene), in which ground state hole carriers, created by doping with a minor additive component, were mainly at an orbital energy set below the hole energy of the major component of a blend [9]. Similarly, the observed phenomena in our system can be explained using the electronic band structure of $\mathrm{PC}_{70} \mathrm{BM}$ and the position of the Fermi level in the density of states of the composition consisting of $\mathrm{PC}_{70} \mathrm{BM}$ and trapped solvent molecules. Energy levels of such molecular semiconductors are determined primarily by molecular orbitals, and their energy can be derived electrochemically, spectroscopically, or by means of semi-empirical calculations. Gao et al. have proposed a numerical model applicable to doped organic materials showing that the Seebeck coefficient increases as greater proportions of the electrical conductivity occur at energies different from the Fermi level [20]. This depends on the existence of states away from the Fermi level, the probability of their occupancy, and the charge carrier mobility in these states. Wuesten et al. extended the model used by Gao et al. allowing hopping transport and the effects of defects typical for organics, such as grain boundaries, that produce additional localized states [21]. Both models predict the experimental behavior of a variety of organic materials showing that high doping levels decrease $S$ because the Fermi level is brought closer to the energy level where charge transport is favored. The above-mentioned models can be applied to our preliminary results regarding the establishment of a Fermi level with respect to the orbital energies of the two molecular components. In our system of two components, the conductive molecular semiconductor $\mathrm{PC}_{70} \mathrm{BM}$ as a main component has carrier energies just above the orbital energies of the trapped molecules of the added solvent that form the minor component. The selected solvents exhibit different molecular structures, polarity, and redox potential with respect to $\mathrm{PC}_{70} \mathrm{BM}$. The Fermi level is established by the additive, while the current from injected charge is carried predominantly in the higher energy orbitals of the bulk composition. Hence, thermal excitation of additive-generated charge carriers in one region of the sample can lead to the migration of some of them into the bulk energy levels of another region of the sample, increasing thermoelectric voltage. On the other hand, the Seebeck coefficient increases in the presence of a strong phonon-electron coupling and counteracts the effects of the Seebeck coefficient decrease caused by enhanced charge carrier mobility [22,23]. However, these hypotheses are only speculations and require further study.

To find out how the mixed solvents affect the morphology of the $\mathrm{PC}_{70} \mathrm{BM}$ samples, we investigated the sample surface by using an atomic force microscope (AFM). Figure 7 shows the corresponding AFM images. The sample morphology of the prepared $\mathrm{PC}_{70} \mathrm{BM}$ films does not significantly change. However, the surface roughness slightly decreases when the second solvent is added, indicating that more compact and ordered structures are formed, which could imply higher charge carrier mobility.

The root-mean-square (RMS) surface roughness was found to be $0.72,0.62,0.45,0.37,0.35$, and $0.3 \mathrm{~nm}$ for pristine, CB:DCE, CB:AC, CB:DMF, CB:NMP and CB:DMSO samples, respectively.

Figure 8 shows the thermoelectric power factor of our samples calculated using $P=S^{2} \sigma$. The highest power factor was obtained from the DMSO sample, which shows that among these solvents, DMSO is the best one for preparing PCBM solutions to obtain samples with a higher thermoelectric power factor. 


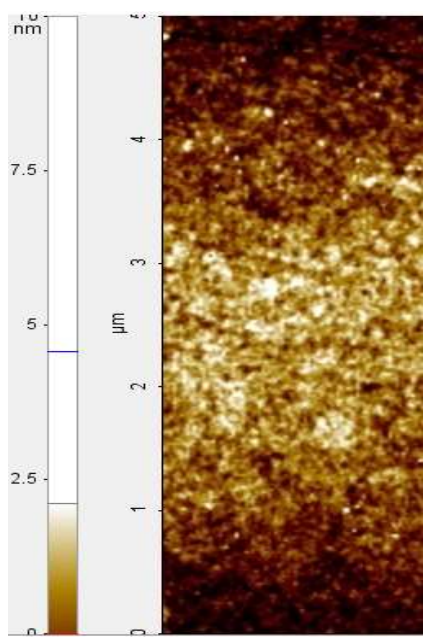

(a)

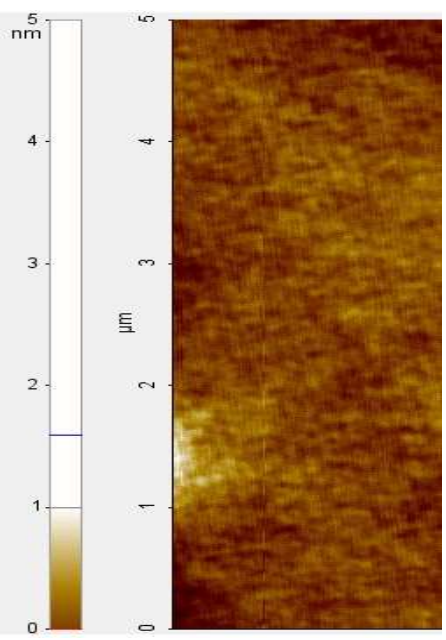

(d)

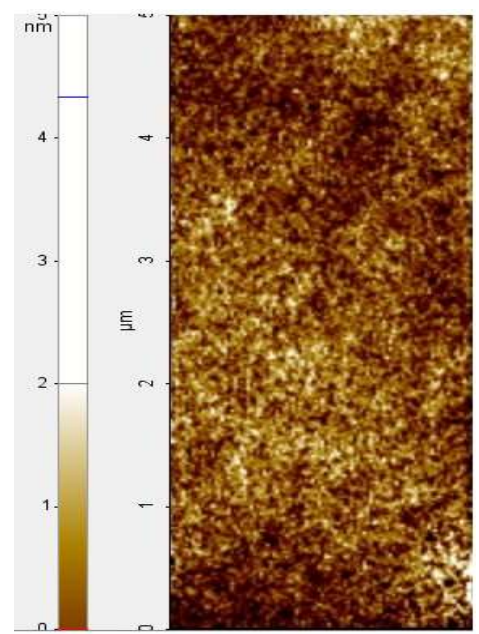

(b)

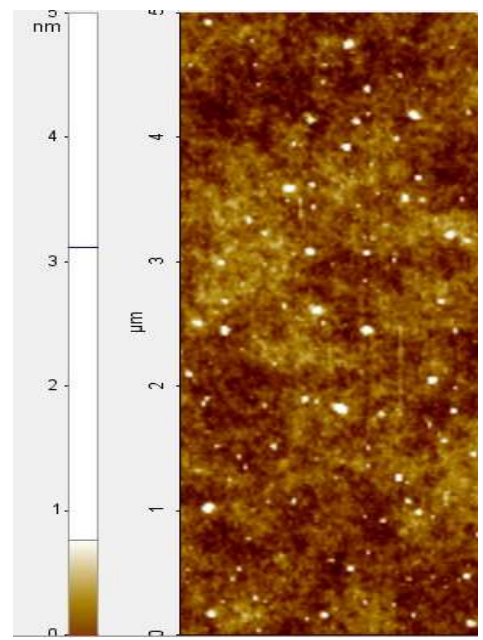

(e)

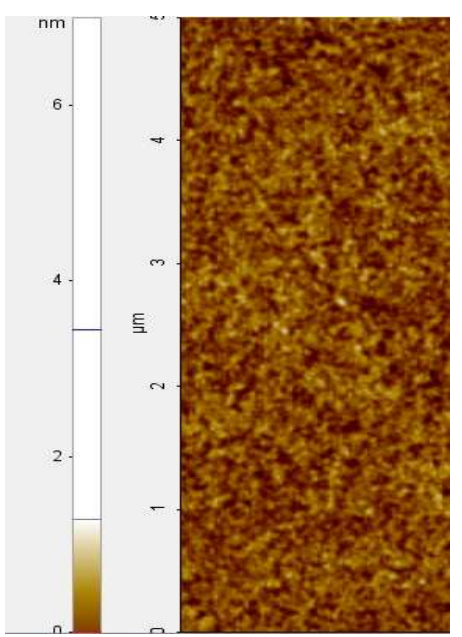

(c)

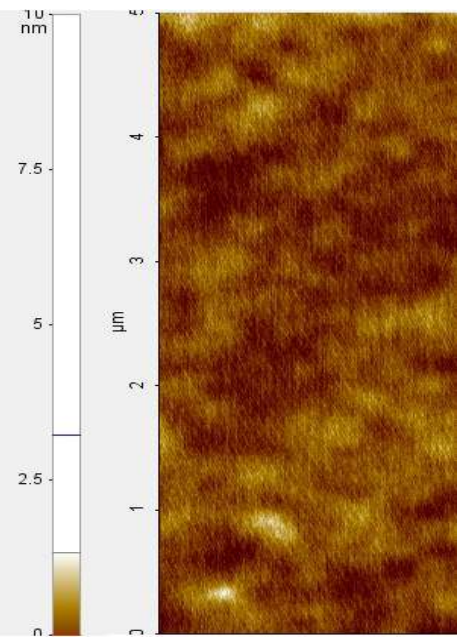

(f)

Figure 7. AFM images of the samples. (a) CB; (b) CB:DCE; (c) CB:AC; (d) CB:DMF; (e) CB:NMP; (f) CB:DMSO.

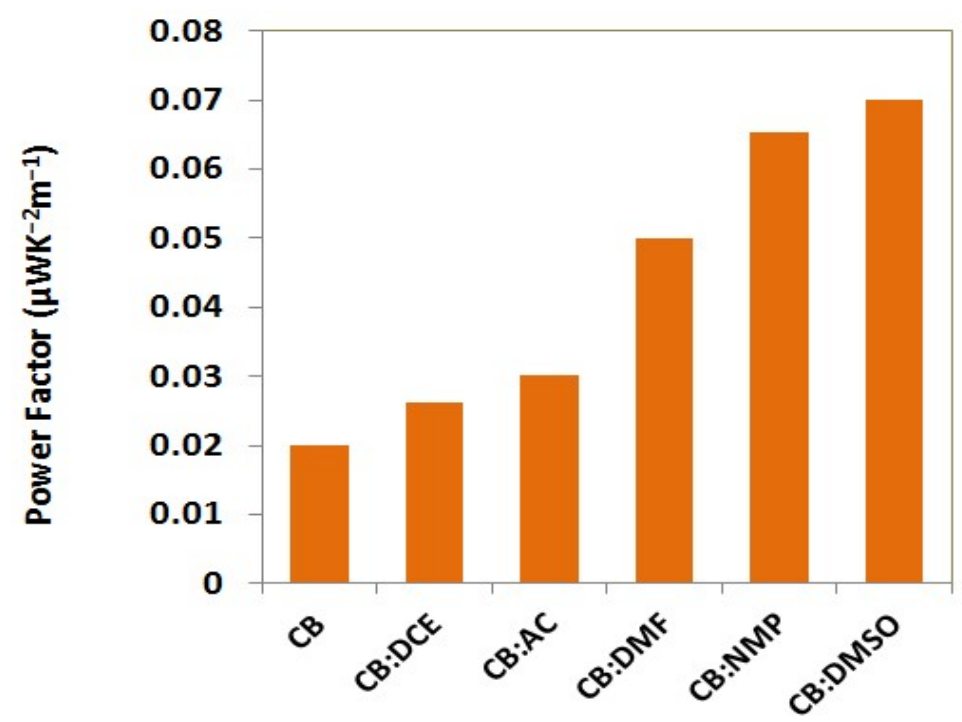

Figure 8. Thermoelectric power factor values of pristine and treated PCBM. 
The calculated values of the power factor are 0.02, 0.026, 0.030, 0.05, 0.065, and $0.07 \mu \mathrm{WK}^{-2} \mathrm{~m}^{-1}$ for pristine, CB:DCE, CB:AC, CB:DMF, CB:NMP, and CB:DMSO, respectively. It is worth noting that the power factor value of $\mathrm{PC}_{70} \mathrm{BM}$ is higher than a variety of conducting polymers, including poly(3,4-ethylenedioxythiophene) polystyrene sulfonate (PEDOT/PSS), poly [N-90-heptadecanyl-2,7-carbazole-alt-5,5-(40,70-di-2-thienyl-20,10,3-benzothiadizole)] (PCDTBT), poly(paraphenylene), poly(p-phenylenevinylene), poly(carbazolenevinylene) derivative, and polyaniline $[10,11,14,15]$.

\section{Conclusions}

Using the procedure of mixed-solvents for the $\mathrm{PC}_{70} \mathrm{BM}$, we have demonstrated that both thermoelectric parameters, electrical conductivity, and the Seebeck coefficient can be manipulated in such a way that a simultaneous enhancement of both factors can be achieved. Due to the entrapped molecules of solvents in the PCBM network, an increase in the charge carrier mobility and the Seebeck coefficient was possible during maintaining a constant number of charge carriers. The simultaneous enhancement in electrical conductivity and the Seebeck coefficient closely correlates with the experimentally determined charge carrier mobility. From a series of investigated solvents, DMSO and NMP with higher boiling points appeared to be the most effective co-solvents in improving the power factor.

Author Contributions: M.R. conceived, designed, and performed the experiments; U.D.-W. coordinated scientific work and interpreted the experimental results; and C.L. wrote the paper. All authors read and approved the final version of the manuscript to be submitted.

Acknowledgments: This work was supported by the National Research Foundation of Korea (NRF-2016R1A2B3009301) and LG Chem, Ltd., Korea.

Conflicts of Interest: The authors declare no conflicts of interest.

\section{References}

1. Poudel, B.; Hao, Q.; Ma, Y.; Lan, Y.; Minnich, A.; Yu, B.; Yan, X.; Wang, D.; Muto, A.; Vashaee, D.; et al. High-Thermoelectric Performance of Nanostructured Bismuth Antimony Telluride Bulk Alloys. Science 2008, 320, 634-638. [CrossRef] [PubMed]

2. Zhao, W.; Fan, S.; Xiao, N.; Liu, D.; Tay, Y.Y.; Yu, C.; Sim, D.; Hng, H.H.; Zhang, Q.; Boey, F.; et al. Flexible Carbon Nanotube Papers with Improved Thermoelectric Properties. Energy Environ. Sci. 2012, 5, 5364-5369. [CrossRef]

3. Culebras, M.; Gómez, C.M.; Cantarero, A. Thermoelectric measurements of PEDOT:PSS/expanded graphite composites. J. Mater. Sci. 2013, 48, 2855-2860. [CrossRef]

4. Dubey, N.; Leclerc, M. Conducting Polymers: Efficient Thermoelectric Materials. J. Polym. Sci. B Polym. Phys. 2011, 49, 467-475. [CrossRef]

5. Bubnova, O.; Khan, Z.U.; Malti, A.; Braun, S.; Fahlman, M.; Berggren, M.; Crispin, X. Optimization of the thermoelectric figure of merit in the conducting polymer poly(3,4-ethylenedioxythiophene). Nat. Mater. 2011, 10, 429-433. [CrossRef] [PubMed]

6. Zhang, Q.; Sun, Y.M.; Xu, W.; Zhu, D.B. Thermoelectric energy from flexible P3HT films doped with a ferric salt of triflimide anions. Energy Environ. Sci. 2012, 5, 9639-9644. [CrossRef]

7. Gao, F.; Liu, Y.; Xiong, Y.; Wu, P.; Hu, B.; Xu, L. Fabricate organic thermoelectric modules use modified PCBM and PEDOT:PSS materials. Front. Optoelectron. 2017, 10, 117-123. [CrossRef]

8. Petsagkourakis, I.; Pavlopoulou, E.; Portale, G.; Kuropatwa, B.A.; Dilhaire, S.; Fleury, G.; Hadziioannou, G. Structurally-driven enhancement of thermoelectric properties within poly(3,4-ethylenedioxythiophene) thin films. Sci. Rep. 2016, 6, 30501. [CrossRef] [PubMed]

9. Sun, J.; Yeh, M.L.; Jung, B.J.; Zhang, B.; Feser, J.; Majumdar, A.; Katz, H.E. Simultaneous increase in Seebeck coefficient and conductivity in a doped poly(alkylthiophene) blend with defined density of states. Macromolecules 2010, 43, 2897-2903. [CrossRef] 
10. Maiz, J.; Rojo, M.M.; Abad, B.; Wilson, A.A.; Nogales, A.; Borca-Tasciuc, D.A.; Borca-Tasciuc, T.; Martín-González, M. Enhancement of thermoelectric efficiency of doped PCDTBT polymer films. RSC Adv. 2015, 5, 66687-66694. [CrossRef]

11. Xuan, Y.; Liu, X.; Desbief, S.; Leclère, P.; Fahlman, M.; Lazzaroni, R.; Berggren, M.; Cornil, J.; Emin, D.; Crispin, $\mathrm{X}$. Thermoelectric properties of conducting polymers: The case of poly(3-hexylthiophene). Phys. Rev. B 2010, 82, 115454. [CrossRef]

12. Mateeva, N.; Niculescu, H.; Schlenoff, J.; Testardi, L.R. Correlation of Seebeck coefficient and electric conductivity in polyaniline and polypyrrole. J. Appl. Phys. 1998, 83, 3111-3117. [CrossRef]

13. Kaiser, A.B. Thermoelectric power and conductivity of heterogeneous conducting polymers. Phys. Rev. $B$ 1989, 40, 2806. [CrossRef]

14. Toshima, N. Conductive polymers as a new type of thermoelectric material. Macromol. Symp. 2002, 186, 81-86. [CrossRef]

15. Jiang, Q.; Liu, C.; Song, H.; Shi, H.; Yao, Y.; Xu, J.; Zhang, G.; Lu, B. Improved thermoelectric performance of PEDOT:PSS films prepared by polar-solvent vapor annealing method. J. Mater. Sci. Mater. Electron. 2013, 24, 4240-4246. [CrossRef]

16. Ma, W.; Shi, K.; Wu, Y.; Lu, Z.Y.; Liu, H.Y.; Wang, J.Y.; Pei, J. Enhanced molecular packing of a conjugated polymer with high organic thermoelectric power factor. ACS Appl. Mater. Interfaces 2016, 8, 24737-24743. [CrossRef] [PubMed]

17. Rastegaralam, M.; Lee, C.; Dettlaff-Weglikowska, U. Solvent-Dependent Thermoelectric Properties of PTB7 and Effect of 1,8-Diiodooctane Additive. Crystals 2017, 7, 292. [CrossRef]

18. Kim, S.S.; Bae, S.; Jo, W.H. Performance enhancement of planar heterojunction perovskite solar cells by n-doping of the electron transporting layer. Chem. Commun. 2015, 51, 17413-17416. [CrossRef] [PubMed]

19. Wei, P.; Oh, J.H.; Dong, G.; Bao, Z. Use of a $1 H$-Benzoimidazole Derivative as an $n$-Type Dopant and to Enable Air-Stable Solution-Processed n-Channel Organic Thin-Film Transistors. JACS 2010, 132, 8852-8853. [CrossRef] [PubMed]

20. Gao, X.; Uehara, K.; Klug, D.D.; Tse, J.S. Rational design of high-efficiency thermoelectric materials with low band gap conductive polymers. Comput. Mater. Sci. 2006, 36, 49-53. [CrossRef]

21. Wuesten, J.; Ziegler, C.; Ertl, T. Electron transport in pristine and alkali metal doped perylene-3,4,9,10-tetracarboxylicdianhydride (PTCDA) thin films. Phys. Rev. B 2006, 74, 125205. [CrossRef]

22. Heremans, J.P.; Jovovic, V.; Toberer, E.S.; Saramat, A.; Kurosaki, K.; Charoenphakdee, A.; Yamanaka, S.; Snyder, G.J. Enhancement of thermoelectric efficiency in PbTe by distortion of the electronic density of states. Science 2008, 25, 554. [CrossRef] [PubMed]

23. Martin, J.; Wang, L.; Chen, L.D.; Nolas, G.S. Enhanced Seebeck coefficient through energy-barrier scattering in PbTe nanocomposites. Phys. Rev. B 2009, 79, 115311. [CrossRef] 